\title{
Cannabidiol Attenuates Palmitic Acid-Induced Injury in Cultured Hepatocytes Through Promoting Autophagic Flux
}

\author{
Rui Chen ${ }^{1, \dagger}$, Xiaogang Gao ${ }^{1, \dagger}$, Lei Zhang ${ }^{1}$, Wenyu Zhao ${ }^{1}$, Li Zeng ${ }^{1, ~}$, Youhua Zhu ${ }^{1}$, Zhiren Fu ${ }^{2, *}$ \\ ${ }^{1}$ Organ Transplantation Center, Changhai Hospital of Naval Medical University, Shanghai, China \\ ${ }^{2}$ Organ Transplantation Center, Changzheng Hospital of Naval Medical University, Shanghai, China
}

Email address:

Zengli111109@aliyun.com (Li Zeng),Zhirenf@sh163.net (Zhiren Fu)

${ }^{*}$ Corresponding author

$†$ Rui Chen and Xiaogang Gao are co-first authors.

\section{To cite this article:}

Rui Chen, Xiaogang Gao, Lei Zhang, Wenyu Zhao, Li Zeng, Youhua Zhu, Zhiren Fu. Cannabidiol Attenuates Palmitic Acid-Induced Injury in Cultured Hepatocytes Through Promoting Autophagic Flux. International Journal of Clinical and Experimental Medical Sciences. Vol. 4, No. 3, 2018, pp. 51-56. doi: 10.11648/j.ijcems.20180403.15

Received: June 21, 2018; Accepted: August 2, 2018; Published: August 31, 2018

\begin{abstract}
Objective: This work was designed to investigate the protection of cannabidiol (CBD) against palmitic acid (PA)-induced injury in cultured hepatocytes and the underlying mechanism associated with autophagic flux. Methods: Experiment 1: Primary cultured hepatocytes were stimulated with PA $(800 \mu \mathrm{mol} / \mathrm{L})$ and treated with $\mathrm{CBD}(5 \mu \mathrm{mol} / \mathrm{L})$ and chloroquine (CQ, $50 \mathrm{nmol} / \mathrm{L}$ ) or not for 24 hours (1: control group; 2: PA-stimulated group; 3: PA-stimulated group treated with CBD; 4: PA-stimulated group treated with CBD and CQ). Autophagic flux was evaluated by Western blot analysis. Apoptosis was measured by flow cytometry. The mRNA expression of genes involved in endoplasmic reticulum stress was determined by reverse transcription PCR. The mitochondrial function was determined by using fluorescent probe including Rh123 and lucigenin. Experiment 2: Primary cultured hepatocytes were treated with CBD alone for $24 \mathrm{~h}$ (1: control group; 2: lower-dose CBD-treated group; 3: higher-dose CBD-treated group). Then, the autophagic flux was evaluated by Western blot analysis. Results: When compared to control group, exposure to PA significantly led to impaired autopagic flux (evidenced by increased ratio of LC3-II/LC3-I and protein expression of p62), increased apoptosis, endoplasmic reticulum stress (evidenced by increased mRNA expression of C/EBP homologous protein, glucose-regulated protein 78, and X-box protein 1), and mitochondrial dysfunction (evidenced by reduced mitochondrial membrane potential and enhanced formation of mitochondrial reactive oxygen species). When compared to PA-stimulated group, CBD treatment significantly attenuated PA-induced impaired autophagic flux, apoptosis, endoplasmic reticulum stress, and mitochondrial dysfunction in cultured hepatocytes. The protection of CBD against PA was abolished by co-incubation with CQ. In addition, treatment with CBD alone had no significant effect on autophagic flux in cultured hepatocytes Conclusion: Cannabidiol attenuates palmitic acid-induced impaired autophagic flux, apoptosis, endoplasmic reticulum stress, and mitochondrial dysfunction in cultured hepatocytes through promoting autophagic flux.
\end{abstract}

Keywords: Cannabidiol, Palmitic Acid, Autophagic Flux, Hepatocytes, Apoptosis, Mitochondrial Dysfunction, Endoplasmic Reticulum Stress

\section{Introduction}

Nonalcoholic fatty liver disease (non-alcoholic fatty liver disease) refers to the elimination of alcohol, virus and other clear liver damage factors, which are caused by genetic, environmental and metabolic stress related factors that lead to the deposition of lipid in the liver cells, and then lead to diffuse fatty degeneration of the liver cells. The development of the liver fibrosis, liver cirrhosis, and even hepatocellular carcinoma will occur in the later stage. With the improvement of material life, nonalcoholic fatty liver disease is becoming more and more common as a chronic liver disease. It has attracted more and more attention from medical workers [1].

Although the medicinal value of cannabis has long been well known, its clinical application has been greatly restricted because of its addiction and psychogenic hallucination. Extraction found that the main active ingredient in cannabis is 
9-four hydrogen cannabinol and cannabidiol (CBD). In recent years, the pharmacological action of CBD has been paid more and more attention. Unlike Delta 9- four hydrogen cannabinol, CBD has no addiction and psychedelic action [2], which is very beneficial to its clinical application. CBD can play a pharmacological role through the receptor and non receptor, and the current research on CBD mainly focuses on its immune regulation, angiogenesis, anti-cancer and neuroprotective effects of the cardiovascular and other [3]. In the liver, in vitro cell experiments found that the treatment of delta 9-four hydrogen or CBD can reduce oleic acid (unsaturated fatty acid) aggregation in liver cells [4], suggesting that CBD may be used to reduce the damage of fatty acids to liver cells during the pathogenesis of nonalcoholic fatty liver disease. But oleic acid is a kind of unsaturated fatty acid, and its liver toxicity is higher than that of unsaturated fatty acids $[5,6]$. It has been demonstrated that nonalcoholic fatty liver disease is mainly induced by saturated fatty acids and induces apoptosis. [5, 6]. In order to confirm the potential therapeutic effect of CBD in non-alcoholic fatty liver, it is necessary to further observe whether CBD has a therapeutic effect on liver injury induced by saturated fatty acids. Palmitic acid is a saturated fatty acid. It is also used to simulate the pathogenesis of nonalcoholic fatty liver disease in vitro and induce liver cells to damage [7].

The main purpose of this study is to observe whether CBD has a therapeutic effect on the adipose toxicity induced by palmitic acid in liver cells. It also provides a necessary experimental basis for further study of whether CBD can be used in the treatment of nonalcoholic fatty liver disease in the future. This study also preliminarily discussed the protection mechanism of CBD.

\section{Materials and Methods}

\subsection{Experimental Animals}

8 weeks old SD rats (250-300 grams), female and male half, clean grade, purchased from the Second Military Medical University animal experiment centre, the production license number is SCXK (Shanghai 2012-0003), the animal use license number is SYXK (Shanghai 2012-0003).

\subsection{Primary Rat Hepatocytes Culture and Experimental Treatment}

According to the methods described in the literature [8], rats were injected with $3 \%$ pentobarbital sodium $(0.2$ $\mathrm{ml} / 0.1 \mathrm{~kg}$ ) intraperitoneal anesthesia, fixed rats, $75 \%$ alcohol sterilizing the chest and abdomen, $\mathrm{U}$ caesarean section, $18 \mathrm{G}$ indwelling needle puncture portal vein, 37 centigrade Hank 's balance salt solution (American Gibco company) rinsed the liver, and then preheated at 37 centigrade with type IV type Collagenase Hank 's balanced saline solution $(0.75 \mathrm{mg} / \mathrm{mL}, 5$ $\mathrm{ml} / \mathrm{min}$ ) was perfused, and the liver was extracted and shredded in DMEM culture medium at 4 degree pre cooling. The cell suspension was filtered through a $70 \mu \mathrm{m}$ nylon sieve and filtered through a $40 \mathrm{~m}$ nylon sieve. At $4 \mathrm{C}, 30 \mathrm{~g}$ centrifugation $5 \mathrm{~min}$, precipitation was washed two times with DMEM solution. Cell counts, $5 \times 105$ cells $/ \mathrm{mL}(10 \%$ peptide bovine serum DMEM, fetal bovine serum, American Gibco company, 30044-033) inoculated cells. The cells were incubated in incubators at $37 \mathrm{C}, 5 \% \mathrm{CO} 2$ and $95 \%$ air. After $6 \mathrm{~h}$, the cells adhered to the wall, and the experiment began after $24 \mathrm{~h}$.

\subsection{Experimental Design}

Experiment 1: (1) control group; (2) palmitic acid (palmitic acid, PA, $800 \mathrm{mu} \mathrm{mol} / \mathrm{L}$, American Sigma company, cargo number P0500) stimulation group; (3) PA (800 mu mol/L) +CBD ( 5 mu mol/L, American Sigma company, cargo number C7515) group; (800 micron) (5 mu) + chloroquine Division, cargo number C6628) treatment group. The specific methods are as follows: PA, CBD and CQ are dissolved in DMSO before the experiment, and the dissolution concentration is 10000 times the concentration. The original solution was added to the DMEM medium at the same time, then added blank DMSO to complement (the first three groups) and mix well. Finally, the concentration of DMSO in each group was $0.03 \%$. The multi drug treatment group was added to the medium simultaneously. 3 compound holes were set in each experiment group. The cells were adhered to $24 \mathrm{~h}$ and began to experiment. The cells were washed with serum-free DMEM for two times, then the DMEM medium containing drugs was added, and the drug treatment time was $24 \mathrm{~h}$.

Experiment 2: (1) control group; (2) CBD (1 mol/L) group; and (3) CBD (5 mol/L) group. The degree of autophagy was examined. The specific treatment is: the dissolution process of the drug is the same as above, and each experimental group has 3 holes. The cells were adhered to $24 \mathrm{~h}$ and began to experiment. The cells were washed with serum-free DMEM for two times, then the DMEM medium containing drugs was added, and the drug treatment time was $24 \mathrm{~h}$.

\subsection{Western Blot}

Add Western and IP cell lysate (Shanghai Biyun Tian biological reagent company, P0013) to collect cell protein, $100 \mathrm{C}, 10 \mathrm{~min}$ protein denaturation, use the protein concentration quantitative reagent box (Shanghai gmei gene medicine science and technology, cargo number GMS30031) to detect protein concentration, SDS-PAGE gel electrophoresis separation protein, wet transfer method of protein Transfer to PVDF film, 5\% skim milk was closed at room temperature for $4 \mathrm{~h}$. After TBST solution was washed, one anti and two anti solution was added, and ECL color reagent box (Shanghai Biyun Tian biological reagent company, freight number P0018A) was added to the chromogenic substrate reaction, film compression film was developed, and Image $\mathrm{J}$ image analysis software was used to analyze the gray value of strip, GAPDH protein (G) APDH protein antibody, Santa Cruz, SC-32233) is an internal reference protein, the target protein is LC3-I, LC3-II (LC3 antibody, American Sigma company, cargo number L7543, used for LC3-I and LC3-II) and p62. 


\subsection{Detection of Apoptosis by Flow Cytometry}

According to the instructions of the cell apoptosis detection kit (Invitrogen company, V13241), the cells were collected, $800 \mathrm{~g}$ centrifuged $8 \mathrm{~min}$, and PBS was washed two times. Annexin V (Annexin V) and Propidium Iodide (PI) were added to blend and then incubated for 15 min under the condition of 25 degrees centigrade. Cell apoptosis was detected by flow cytometry, and the proportion of apoptotic cells was calculated.

\subsection{Real-Time Quantitative PCR Was Used to Detect the mRNA Level of Endoplasmic Reticulum Stress Related Genes}

Remove the medium, wash PBS solution for 2 times, add 500 $\mu 1$ Trizol solution per hole, extract the total RNA of the cell. Random primers reverse transcription of single strand DNA, and operate the CHOP (5 '-GCA TGA AGG AGA AGG AGC AG-3') according to the quantitative PCR Kit (German QIAGEN company, cargo No. 210210). And the expression level of XBP-1 (5 '-CCT GAG CCC GGA GGA GAA-3'; 5 '-CTC GAG CAG TCT TCT GAG'). MJ opticon monitor software analyses the data obtained. The level of gene expression is expressed by multiplier variation $\left(2^{-\Delta \Delta \mathrm{Ct}}\right.$ Delta $\mathrm{Ct}$ method). Using GAPDH (5 '-AGA CAG CCG CAT CTT CTT GT-3'; 5 '-CTT GCC GTG GGT)) as the internal reference gene; the final value is corrected and then expressed as a multiple of the control group. The primers were synthesized by the bioengineering Engineering (Shanghai) Limited by Share Ltd.

\subsection{Detection of Mitochondrial Function by Probe Method}

The cells were collected and added to the precooled extract $(0.25 \mathrm{~mol} / \mathrm{L}$ of sucrose, $0.01 \mathrm{~mol} / \mathrm{L}$ Tris-HCI, $0.5 \mathrm{mmol} / \mathrm{L}$ EDTA, $0.1 \%$ bovine serum albumin, $\mathrm{pH}$ adjusted to 7.4 ). Differential centrifugation was used to isolate and purify mitochondria. The mitochondrial membrane potential was detected by Rh123 probe (Shanghai Tongren Institute of chemistry, R233) and Ex/Em was 488/525 nm. Lucigenin
(Guangzhou Bao Bo, ENZ-52154) was used as a probe to detect the rate of active oxygen production in mitochondria, and $\mathrm{Ex} / \mathrm{Em}$ was $455 / 505 \mathrm{~nm} .1 .8$ Statistics

\subsection{Statistics}

All data are represented by mean standard deviation. SPSS 12 statistical analysis software was used to perform single factor analysis of variance (One-Way ANOVA) in multiple groups. LSD method was used to test the homogeneity of variance when multiple comparisons were compared, while Dunnett's T3 was used to test the variance when the variance was not homogeneous. When the $\mathrm{P}$ value is less than 0.05 , there is a significant difference between the groups.

\section{Result}

\subsection{In PA Stimulated Hepatocytes, CBD Can Promote Autophagy by Promoting the Degradation of Autophagic Lysosome}

The primary cultured hepatocytes stimulated PA, which could increase the ratio of LC3-II/LC3-I in the liver cells and the protein expression of p62. It was suggested that under the stimulation of PA, the activity of autophagic lysosome increased, but the rate of degradation was reduced and the autophagic flow was destroyed, and the ratio of LC3-II/LC3-I in hepatocytes and the protein expression of P62 could be reduced by $\mathrm{CBD}$ treatment, suggesting that $\mathrm{CBD}$ can promote autophagy flow by promoting autophagic lysosome degradation. In addition, the promoting effect of CBD on autophagic flow was reversed by autophagic inhibitor CQ (Figure 1A, B). Using different doses of CBD alone to treat hepatocytes, it was found that CBD slightly reduced the ratio of LC3-II/LC3-I, but there was no statistical difference. Treatment of hepatocytes with different doses of CBD alone did not significantly affect the protein expression of p62 (Figure 1C, D).
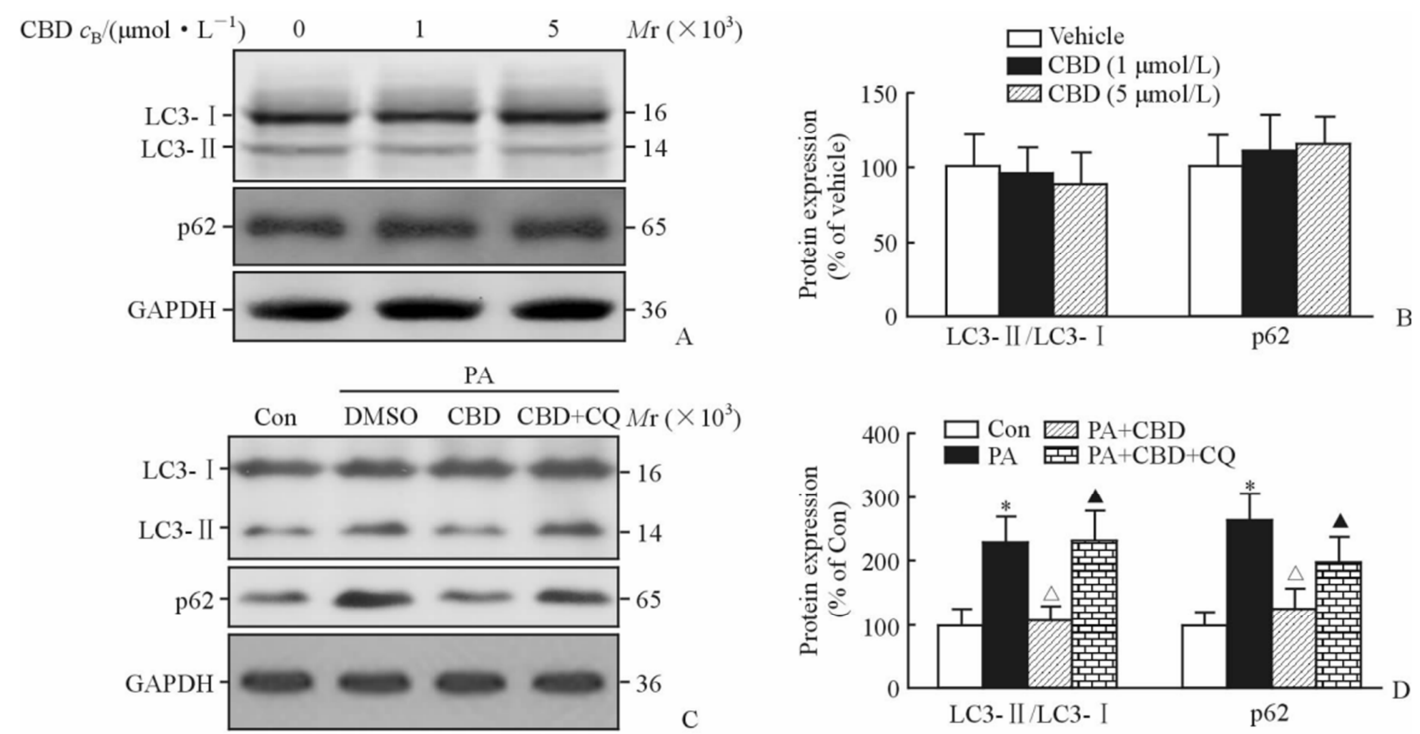

Figure 1. Promoting effect of treatment with $C B D$ on autophagic flux in cultured hepatocytes. $C B D$, Cannabidiol; $P A$, palmitic acid; $C Q$, chloroquine; $* P<0.05$ versus Control group; $\triangle P<0.05$ versus $P A$ group; $\Delta P<0.05$ versus $P A+C B D$ group. 


\subsection{In PA Stimulated Hepatocytes, CBD Alleviates Cell Apoptosis}

Primary cultured hepatocytes were stimulated by PA, which could increase the apoptosis of hepatocytes; CBD treatment could reduce the number of apoptotic cells; at the same time, the autophagic inhibitor CQ stimulation could reverse the therapeutic effect of CBD (Figure 2, the number of cells in Q2 and Q3 quadrants was recorded as the number of apoptotic cells). The results suggest that CBD can reduce PA induced hepatocyte apoptosis by promoting autophagy flow.
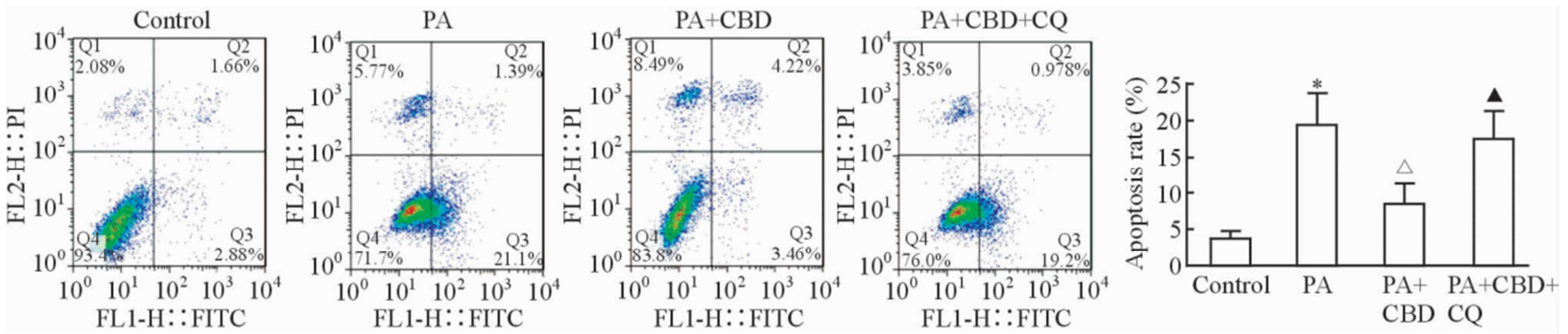

Figure 2. CBD treatment attenuated PA-induced hepatocyte apoptosis in cultured hepatocytes. $C B D$, Cannabidiol; $P A$, palmitic acid; $C Q$, chloroquine; ${ }^{*} P<0.05$ versus Control group; $\triangle P<0.05$ versus $P A$ group; $\boldsymbol{\Delta} P<0.05$ versus $P A+C B D$ group.

\subsection{In PA Stimulated Hepatocytes, CBD Alleviates Endoplasmic Reticulum Stress}

The primary cultured hepatocytes were stimulated by PA, which could increase the mRNA level of CHOP、GRP78 and XBP-1 in liver cells (Figure 3), suggesting that PA induced endoplasmic reticulum stress in liver cells. CBD treatment can reduce the mRNA level of CHOP, GRP78 and XBP-1, while CQ stimulation can reverse the effect of CBD. The results suggest that $\mathrm{CBD}$ can alleviate the $\mathrm{PA}$ induced endoplasmic reticulum stress by promoting the occurrence of autophagic flow.
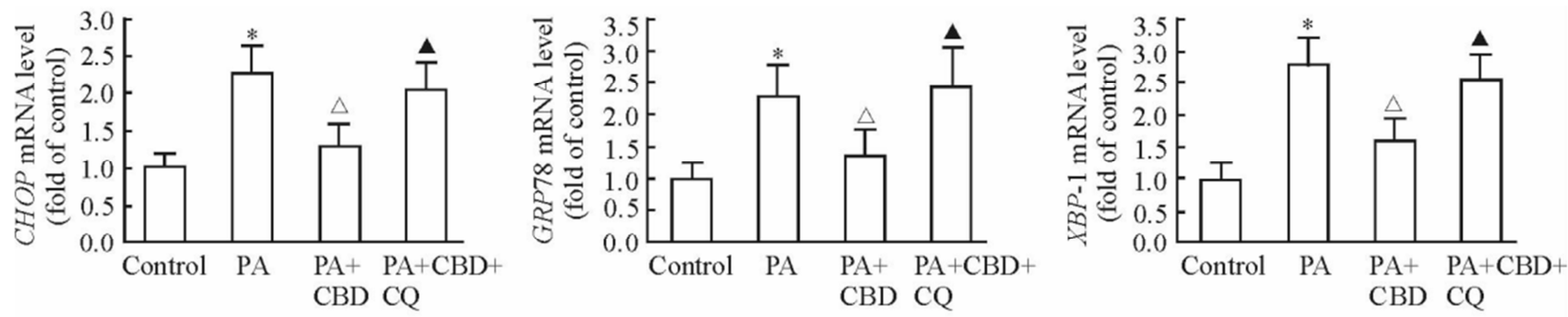

Figure 3. $C B D$ treatment attenuated $P A$-induced endoplasmic reticulum stress in cultured hepatocytes. $C B D$, Cannabidiol; $P A$, palmitic acid; $C Q$, chloroquine; CHOP, C/EBP homologous protein; GRP78, glucose-regulated protein $78 ; X B P-1, X$-box protein $1 ;{ }^{*} P<0.05$ versus Control group; $\triangle P<0.05$ versus $P A$ group; $\Delta P<0.05$ versus $P A+C B D$ group.

\subsection{In PA Stimulated Hepatocytes, CBD Alleviates Mitochondrial Dysfunction}

The primary cultured hepatocytes were stimulated by PA. The mitochondrial membrane potential in hepatocytes decreased (Figure 4A, B), and mitochondrial reactive oxygen species (Figure 4C) increased significantly, suggesting that PA induced the decrease of mitochondrial function in hepatocytes. CBD treatment can increase the mitochondrial membrane potential and reduce the formation of mitochondrial reactive oxygen species. At the same time, CQ stimulation can reverse the protective effect of $\mathrm{CBD}$ on mitochondria, suggesting that CBD can reduce mitochondrial dysfunction in $\mathrm{PA}$ induced hepatocytes by promoting the occurrence of autophagic flow.
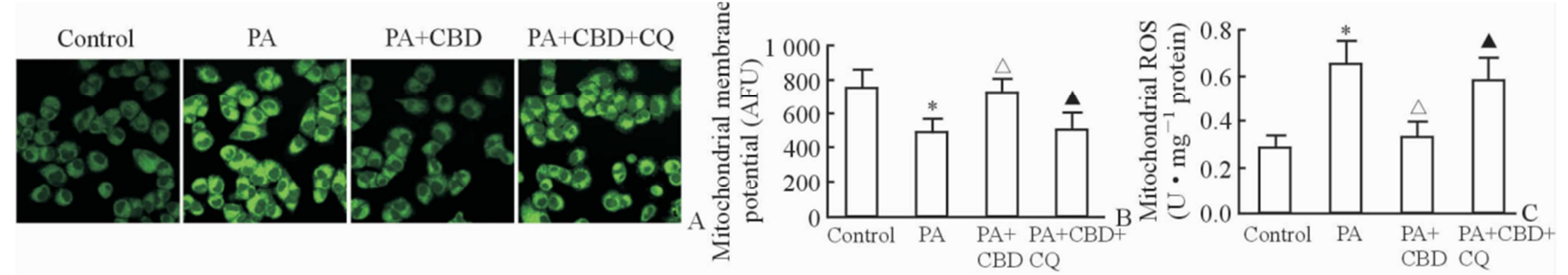

Figure 4. CBD treatment attenuated PA-induced mitochondrial dysfunction in cultured hepatocytes. AFU, arbitrary fluorescence units; ROS, reactive oxygen species; $C B D$, Cannabidiol; $P A$, palmitic acid; $C Q$, chloroquine; $* P<0.05$ versus Control group; $\triangle P<0.05$ versus $P A$ group; $\mathbf{\Delta} P<0.05$ versus $P A+C B D$ group. 


\section{Discussion}

Under stress status, cell autophagy scavenges useless, redundant, damaged or cancerous organelles, abnormal proteins, and lipid droplets, which play an important role in regulating metabolic levels and maintaining the homeostasis of the body's environment. The previous study of autophagy was mostly based on the degree of autophagic evaluation of autophagy, the evaluation of autophagy by the ratio of LC3-II/LC3-I; the increase in autophagy recently found that the level of autophagy did not essentially reflect the level of autophagy, since the number of autophagic bodies was influenced by two aspects of formation and clearance from the angle of autophagic flow. It is true that autophagy includes not only the detection of autophago, but also the dynamic observation of the process of the entire autophagic flow, and the degradation of autophagic substrate p62 is more important for the evaluation of autophagic flow [10]. When the autophagic formation increases, but the autophagic flow is destroyed, the level of autophagic degradation and the accumulation of autophagic bodies in the cells will deteriorate the apoptosis of [11]. Under PA stimulation, although the activity of autophagic lysosome increased in hepatocytes, the rate of degradation and autophagic flow were destroyed, and CBD treatment could reduce the ratio of LC3-II/LC3-I in the liver cells and the protein expression of p62, suggesting that CBD may restore the autophagic flow destroyed by $\mathrm{PA}$ by promoting the degradation of autophagosomes. In addition, CBD has no significant influence on the ratio of LC3-II/LC3-I and the protein expression of p62 in normal liver cells, suggesting that CBD has no effect on the production of autophagic and autophagic flow under the condition of no fatty acid stimulation, and the autophagy level of the cell itself is one of the reasons for this phenomenon in a lower level. The promotion of CBD to autophagy flow has also been reported in other aspects. In the tau disease mouse model, CBD treatment can increase the occurrence of autophagy flow, reduce the deposition of tau protein in the body, thereby alleviating the symptoms of [12]. In the chronic phase of seizure induced by pilocarpine, CBD can promote the occurrence of autophagic flow, thereby reducing the [13] of epilepsy. In alcohol induced fatty liver of mice, CBD also promotes the occurrence of autophagic flow in the liver, thereby alleviating liver injury [14]. Previous studies in the laboratory have found that the promotion of autophagic flow not only reduces the lipid deposition in the liver cells, but also reduces endoplasmic reticulum stress and mitochondrial damage, thus alleviating the fatty liver disease [15] induced by methionine / choline deficiency diet in mice, which suggests that the recovery of PA destroyed autophagic flow for CBD alleviates PA Induced hepatocyte injury may be very important.

The level of endoplasmic reticulum stress in liver cells was upregulated by PA stimulation. Endoplasmic reticulum (ER) is the main place for protein synthesis, folding, maturation and secretion in cells. In the condition of disease, the protein is not normally modified by some factors or the protein is blocked by the endoplasmic reticulum to the Golgi body, causing unfolded protein or error folded protein to accumulate in the endoplasmic reticulum, and then destroy the balance of calcium ions, resulting in a typical endoplasmic reticulum stress. There are plasma protein synthesis and secretion, lipoprotein and extremely low density lipoprotein synthesis and secretion, cholesterol synthesis and exogenous metabolism, and metabolism is very active in the liver cells. In non-alcoholic steatohepatitis, endoplasmic reticulum stress participates in and promotes the occurrence and development of the disease, and promotes the [16] of fat deposition, inflammatory response, oxidative stress and apoptosis in the liver cells. Autophagy agonist treatment can alleviate the [8] induced by $\mathrm{PA}$ in the endoplasmic reticulum stress of hepatocytes. Therefore, CBD can alleviate PA induced endoplasmic reticulum stress by promoting autophagy flow.

Under the stimulation of PA, the mitochondrial membrane potential of hepatocytes decreased, and the production of mitochondrial reactive oxygen species increased. No matter what the initial cause is, mitochondrial dysfunction plays an important role in nonalcoholic steatohepatitis, [17]. Autophagy maintains the number and health of mitochondria in cells by clearing too many or damaged mitochondria. Mitochondria are the main organs of active oxygen generation in cells. When mitochondria damage, the membrane potential decreases, membrane permeability increases, and the release of reactive oxygen is increased. At the same time, the release of cytochrome $\mathrm{c}$ and other factors into the cytoplasm can induce mitochondrial apoptosis, and autophagy can reduce the occurrence of [18]. In HepG2 hepatocytes, mitochondrial damage induced by peanut four enoic acid, sulfoxide or chloroform can be alleviated by autophagy agonist [19]. Therefore, CBD can reduce the mitochondrial damage induced by $\mathrm{PA}$ and reduce mitochondrial related oxidative stress and apoptosis by promoting autophagy flow.

\section{Conclusion}

In conclusion, CBD alleviated palmitic acid induced hepatocyte apoptosis, endoplasmic reticulum stress and mitochondrial dysfunction through promoting autophagy flow.

Previous animal study have shown that CBD attenuated nonalcoholic steatohepatitis (NASH) induced by methionine-choline-deficient diet (MCD) in rats [20]. The cell experiment showed that $\mathrm{CBD}$ also has a protective effect on PA induced hepatocyte injury. The animal and cell experiment indicated CBD play in the process of liver protective effect can promote the flow of autophagy, a process that in addition to the regular change of autophagy marker LC3 and P62, application of autophagy strengthening agent and the inhibitor can be observed in NASH and liver cell injury after each relevant links are in the process of the 
corresponding change significantly. This phenomenon suggests that autophagy plays an important role in the process of NASH, which is one of the important mechanisms of cannabidiol's protective effect on NASH.

\section{References}

[1] HUANG X, XU M, CHEN Y, PENG K, HUANG Y, WANG P, et al. Validation of the fatty liver index for nonalcoholic fatty liver disease in middle-aged and elderly Chinese [J]. Medicine (Baltimore), 2015, 94: e1682.

https://www.ncbi.nlm.nih.gov/pmc/articles/PMC4616754/.

[2] JACOBS DS, KOHUT SJ, JIANG S, NIKAS SP,

MAKRIYANNIS A, BERGMAN J. Acute and chronic effects of cannabidiol on $\Delta^{9}$-tetrahydrocannabinol $\left(\Delta^{9}\right.$-THC)-induced disruption in stop signal task performance [J]. Exp Clin Psychopharmacol, 2016, 24:320-330.

http://content.apa.org/journals/pha/24/5/320.

[3] PISANTI S, MALFITANO AM, CIAGLIA E, LAMBERTI A, RANIERI R, CUOMO G, et al. Cannabidiol: State of the art and new challenges for therapeutic applications [J]. Pharmacol Ther, 2017, (in press).

http://www.sciencedirect.com/science/article/pii/S0163725817 300657 .

[4] SILVESTRI C, PARIS D, MARTELLA A, MELCK D, GUADAGNINO I, CAWTHORNE $M$, et al. Two non-psychoactive cannabinoids reduce intracellular lipid levels and inhibit hepatosteatosis [J]. J Hepatol, 2015, 62:1382-1390.

https://linkinghub.elsevier.com/retrieve/pii/S0168-8278 00003-3.

[5] BARREYRO FJ, KOBAYASHI S, BRONK SF, WERNEBURG NW, MALHI H, GORES GJ. Transcriptional regulation of $\mathrm{Bim}$ by FoxO3A mediates hepatocyte lipoapoptosis [J]. J Biol Chem, 2007, 282:27141-27154. http://www.jbc.org/cgi/pmidlookup?view=long\&pmid=17626 006.

[6] MALHI H, BRONK SF, WERNEBURG NW, GORES GJ. Free fatty acids induce JNK-dependent hepatocyte lipoapoptosis [J]. J Biol Chem, 2006, 281:12093-12101. http://www.jbc.org/cgi/pmidlookup?view=long\&pmid=16505 490.

[7] GONZÁLEZ-RODRÍGUEZ A, MAYORAL R, AGRA N, VALDECANTOS MP, PARDO V, MIQUILENA-COLINA $\mathrm{ME}$, et al. Impaired autophagic flux is associated with increased endoplasmic reticulum stress during the development of NAFLD [J]. Cell Death Dis, 2014, 5:e1179. http://dx.doi.org/10.1038/cddis.2014.162.

[8] LIU A, HUANG L, GUO E, LI R, YANG J, LI A, et al. Baicalein pretreatment reduces liver ischemia/reperfusion injury via induction of autophagy in rats [J]. Sci Rep, 2016, 6:25042. http://dx.doi.org/10.1038/srep25042.

[9] ANDING AL, BAEHRECKE EH. Autophagy in cell life and cell death [J]. Curr Top Dev Biol, 2015, 114:67-91. https://linkinghub.elsevier.com/retrieve/pii/S0070-2153 (15) 00048-4.

[10] ZHANG XJ, CHEN S, HUANG KX, LE WD. Why should autophagic flux be assessed [J]? Acta Pharmacol Sin, 2013, 34:595-599.

https://www.ncbi.nlm.nih.gov/pmc/articles/PMC4002868/.
[11] OUYANG L, SHI Z, ZHAO S, WANG FT, ZHOU TT, LIU B, BAO JK. Programmed cell death pathways in cancer: a review of apoptosis, autophagy and programmed necrosis [J]. Cell Prolif, 2012, 45:487-498.

http://onlinelibrary.wiley.com/doi/10.1111/j.1365-2184.2012.0 0845.x/abstract;jsessionid $=6$ A05E1100427B10093982C7765 C242CF.f03t04.

[12] CASAREJOS MJ, PERUCHO J, GOMEZ A, MUÑOZ MP, FERNANDEZ-ESTEVEZ M, SAGREDO O, et al. Natural cannabinoids improve dopamine neurotransmission and tau and amyloid pathology in a mouse model of tauopathy [J]. J Alzheimers Dis, 2013, 35:525-539.

http://content.iospress.com/openurl?genre=article\&id=doi:10. 3233/JAD-130050.

[13] HOSSEINZADEH M, NIKSERESHT S, KHODAGHOLI F, NADERI N, MAGHSOUDI N. Cannabidiol post-treatment alleviates rat epileptic-related behaviors and activates hippocampal cell autophagy pathway along with antioxidant defense in chronic phase of pilocarpine-induced seizure [J]. J Mol Neurosci, 2016, 58:432-440.

https://dx.doi.org/10.1007/s12031-015-0703-6.

[14] YANG L, ROZENFELD R, WU D, DEVI LA, ZHANG Z, CEDERBAUM A. Cannabidiol protects liver from binge alcohol-induced steatosis by mechanisms including inhibition of oxidative stress and increase in autophagy [J]. Free Radic Biol Med, 2014, 68:260-267.

https://linkinghub.elsevier.com/retrieve/pii/S0891-5849 (13) 01567-0.

[15] CHEN R, WANG Q, SONG S, LIU F, HE B, GAO X. Protective role of autophagy in methionine-choline deficient diet-induced advanced nonalcoholic steatohepatitis in mice $[\mathrm{J}]$ Eur J Pharmacol, 2016, 770:126-133.

https://linkinghub.elsevier.com/retrieve/pii/S0014-2999 (15) 30350-2.

[16] BOZAYKUT P, SAHIN A, KARADEMIR B, OZER NK. Endoplasmic reticulum stress related molecular mechanisms in nonalcoholic steatohepatitis [J]. Mech Ageing Dev, 2016, 157:17-29.

https://linkinghub.elsevier.com/retrieve/pii/S0047-6374 (16) 30102-6.

[17] CABRÉ N, CAMPS J, JOVEN J. Inflammation, mitochondrial metabolism and nutrition: the multi-faceted progression of non-alcoholic fatty liver disease to hepatocellular carcinoma [J]. Hepatobiliary Surg Nutr, 2016, 5:438-443. http://dx.doi.org/10.21037/hbsn.2016.09.11.

[18] LIU K, ZHAO Q, LIU P, CAO J, GONG J, WANG C, et al. ATG3-dependent autophagy mediates mitochondrial homeostasis in pluripotency acquirement and maintenance $[\mathrm{J}]$. Autophagy, 2016, 12:2000-2008. http://www.tandfonline.com/doi/full/10.1080/15548627.2016. 1212786 .

[19] WU D, CEDERBAUM AI. Inhibition of autophagy promotes CYP2E1-dependent toxicity in HepG2 cells via elevated oxidative stress, mitochondria dysfunction and activation of p38 and JNK MAPK [J]. Redox Biol, 2013, 1:552-565. https://linkinghub.elsevier.com/retrieve/pii/S2213-2317 00077-3.

[20] CHEN R, GAO XG, ZHANG L, SHI XM, GUO WY, ZHU $\mathrm{YH}$, et al. Therapeutic effect and mechanism of cannabidiol on nonalcoholic fatty liver disease in rats $[\mathrm{J}]$. Med J Chin PLA, 2017, 42:515-519. 